nervous system. In Pediatric Neurology, Ed. Millichap JG. Ped Clin N Amer 1967;14:865$880)$.

\title{
RATE OF DEVELOPMENT OF INTRACTABLE EPILEPSY
}

The time taken to develop intractability of epilepsy was determined prospectively in a cohort of 613 children followed in the Connecticut Study for a median of 9.7 years and reported by researchers from Northern Illinois University, DeKalb, IL; University of California Los Angeles, CA; Yale Medical School, New Haven, CT; and Albert Einstein College of Medicine, Bronx, NY. Intractability was defined in two ways: 1) 2 drugs failed, 1 seizure/month (average) for 18 months (stringent definition), and 2) failure of 2 drugs. Delayed intractability was defined as 3 or more years after epilepsy diagnosis. Intractability (stringent form) developed in 83 children (13.8\%), and the 2-drug definition of intractability was met by $142(23.2 \%)$. Intractability was delayed in $26(31.3 \%)$ children meeting stringent and in $39(27.5 \%)$ meeting the 2-drug definition. Intractability varied with the epilepsy syndrome and was delayed more often in focal than catastrophic (including encephalopathic) epilepsies (stringent: 46.2 vs $14.3 \%, p=0.003$; 2-drug: 40.3 vs $2.2 \%, p<0.0001$ ). Early remission preceded delayed intractability in 65.4 to $74.3 \%$ of cases. After developing intractability, $20.5 \%$ entered remission, and $13.3 \%$ were seizure-free at last follow-up. Referral to surgery may be delayed for 20 or more years, because of this interim period of remission. (Berg AT, Vickrey BG, Testa FM, et al. How long does it take for epilepsy to become intractable? A prospective investigation. Ann Neurol June 2006;60:73-79). (Respond: Anne T Berg PhD, Department of Biology, NIU, DeKalb, IL 60115).

COMMENT. In the earlier Dutch study of 453 children with newly diagnosed epilepsy followed prospectively for 5 years (Arts WFM et al. Brain 2004;127:1774-1784), significant variables for the worst outcome group included a symptomatic or cryptogenic etiology, early age at onset, and a history of febrile seizures. Of $108(24 \%)$ patients with a terminal remission (TS) of $<1$ year, 27 had intractable seizures at 5 years. Of patients receiving 2 or more AEDs, almost $60 \%$ had a TR5 $>1$ year. AEDs were successfully withdrawn in 227 (59\%). The course of epilepsy was constantly favorable in $51 \%$, steadily poor in $17 \%$, remitting after intractability in 25\%, and deteriorating in $6 \%$ (Ped Neur Briefs August 2004;18:57-59).

\section{VALPROATE-INDUCED HYPERAMMONEMIC ENCEPHALOPATHY}

Valproate-induced hyperammonemic encephalopathy (VHE), predisposing causes, clinical, laboratory, and EEG findings, and therapy are reviewed from the Hospital del Mar, Barcelona, Spain. Urea cycle enzyme deficiency, especially ornithine transcarbamylase (OTC), is an inherited cause of hyperammonemia and a risk factor for developing VHE in patients taking VPA. Screening tests are recommended in patients with a known family history of OTC deficiency, and in patients who develop unexplained episodes of confusion, lethargy, and vomiting, and/or increased frequency of seizures, while on VPA therapy. Blood ammonia level, renal and liver function, and urinary orotic acid excretion should be tested. Blood VPA levels are within therapeutic ranges in most cases of VHE, and the dose of VPA and the height of the ammonia level are not related to VHE severity. Organic acidemias 